\title{
Design Principles and Traction Performance of a Novel Zero-spin Rolling Conical Traction Continuously Variable Transmission
}

\section{Chao Li}

Chengdu University of Technology https://orcid.org/0000-0003-2079-076X

\section{Xiuquan Cao}

Sichuan University of Science and Engineering

Qing-tao Li ( $\square$ liqingtao@mail.xhu.edu.cn )

School of Mechanical Engineering, Xihua University https://orcid.org/0000-0003-4376-3078

\section{Original Article}

Keywords: Zero-spin, rolling conical traction CVT, contact ellipse, design principles, efficiency

Posted Date: November 5th, 2020

DOI: https://doi.org/10.21203/rs.3.rs-101796/v1

License: (c) (1) This work is licensed under a Creative Commons Attribution 4.0 International License.

Read Full License 


\section{Title page}

\section{Design Principles and Traction Performance of a Novel Zero-spin Rolling Conical Traction Continuously Variable Transmission}

Chao Li, born in 1989, is currently an associate research fellow at The College of Nuclear Technology and Automation Engineering, Chengdu University of Technology, China. He received his doctor degree on mechanical design and theory from Sichuan University, China, in 2019. His research interests include machine and mechanism theory, robotics and new traction continuously variable transmission technology.

Tel: +86-182-02822945; E-mail: lichao19@cdut.edu.cn

Xiu-quan Cao, born in 1989, is currently a lecturer at Sichuan University of Science and Engineering, China. He received his doctor degree on mechanical design and theory in Sichuan University, China, in 2017.

E-mail: cao_comeon@126.com

Qing-tao Li, born in 1987, is currently an associate professor at School of Mechanical Engineering, Xihua University, China. He received his doctor degree on mechanical design and theory from Sichuan University, China, in 2016. His research interests include hydro-mechainal transmission, machine and mechanism theory, and new traction continuously variable transmission technology.

E-mail: liqingtao@mail.xhu.edu.cn

\section{Corresponding author: Qing-tao Li E-mail: liqingtao@mail.xhu.edu.cn}




\title{
Design Principles and Traction Performance of a Novel Zero-spin Rolling Conical Traction Continuously Variable Transmission
}

\author{
Chao $\mathrm{Li}^{1} \cdot$ Xiu-quan $\mathrm{CaO}^{2} \cdot$ Qing-tao $\mathrm{Li}^{3}$
}

\begin{abstract}
In the existing traction continuously variable transmission (CVT), half toroidal CVT (HT-CVT) is considered to have a better traction performance. However, the HT-CVT has the spin losses due to its structural limitations, which significantly influences the traction efficiency. In this paper, the kinematic qualities and contact models of a novel zero-spin rolling conical CVT (RC-CVT) are studied, and then the rollers and conical disks are compactly designed through the proposed design principles. Subsequently, the transmission efficiency is investigated by using a detailed numerical model and compared with HT-CVT. Based on these computational models and parameters, the practical spin ratio, spin momentum and traction efficiency of RC-CVT are calculated and compared with HT-CVT. The results show that the practical spin ratio and spin momentum of RC-CVT are much smaller than that of HT-CVT, and the efficiency on fixed transmission ratio is consequently higher than that of HT-CVT.
\end{abstract}

Keywords: Zero-spin $\cdot$ rolling conical traction CVT $\bullet$ contact ellipse $\bullet$ design principles $\bullet$ efficiency

\section{Introduction}

Traction continuously variable transmission(CVT) is an ideal mechanical transmission device to reduce vehicle emissions, improve the efficiency of the vehicle, and achieve environmental protection ${ }^{[1,2]}$, because the CVT can provide an infinite number of transmission ratios to allow the engine to operate closer to its optimal efficiency

\section{Qing-tao Li}

liqingtao@mail.xhu.edu.cn

1 The College of Nuclear Technology and Automation Engineering, Chengdu University of Technology, Chengdu 610059, China

2 Sichuan University of Science and Engineering, Zigong, 643002, China

3 School of Mechanical Engineering, Xihua University, Chengdu, 610065, China line ${ }^{[3-5]}$. The main principle of power transmitting of traction CVT is shown in Figure 1. The oil film is created in the contact ellipse between drive component and driven component, and the oil film can become highly viscous (known as elastohydrodynamic state) under the high normal force $\mathrm{F}_{N}$ to ensure the transmitting of a tangential force, which is also called traction force $\mathrm{F}_{T}$. Generally, the traction force $\mathrm{F}_{T}$ can be calculated approximatively by the equation $\mathrm{F}_{T}=\mu \mathrm{F}_{N}$, where the traction coefficient $\mu$ is determined by the rheological characteristics of given oil $^{[6,7]}$.

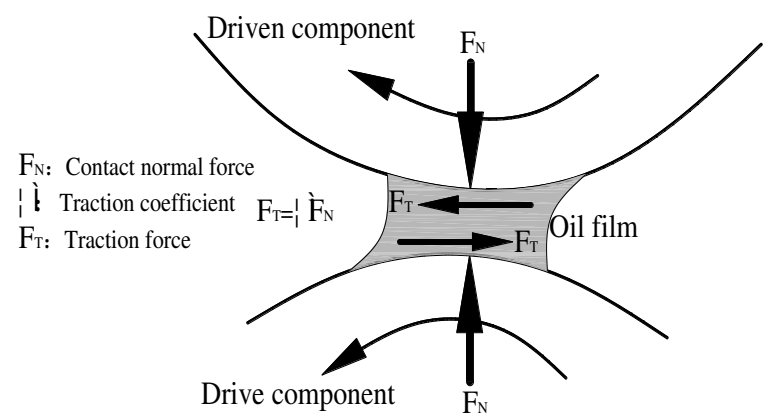

Figure 1 The main principle of power transmitting of t-CVT

Table 1 Category of spin loss

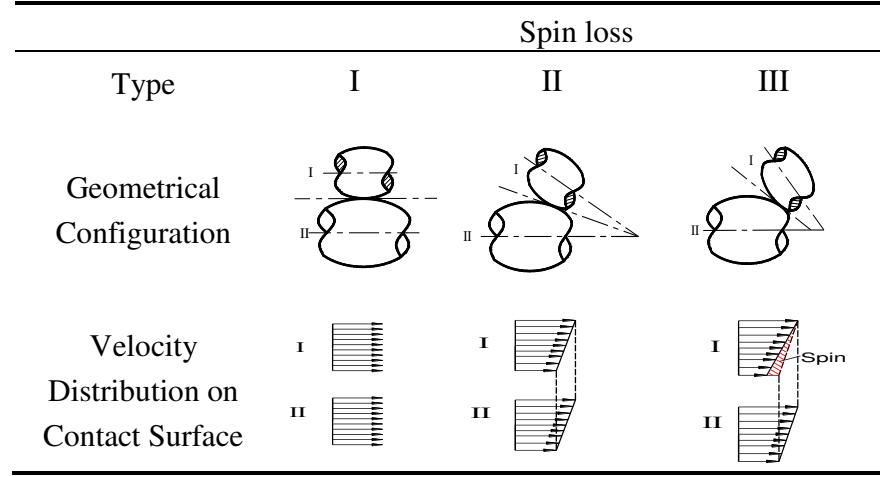


For a traction CVT, there are three main power losses existing in the elastohydrodynamic contact, namely, creep, sideslip and spin. Spin loss is a rotational slip element of the power transmitting contacts, which is determined by a geometrical relation of the input and output rotating components ${ }^{[6,8]}$. A study ${ }^{[6]}$ has described this relationship of spin momentum and geometrical relation, as shown in Table 1 . It is obvious that the rotational axes of two rotating components and the tangential line of the power transmitting contacts are parallel or intersecting at one point, spin is eliminated. Therefore, this special condition is always called zero-spin condition. A large number of studies ${ }^{[9-12]}$ have shown that spin losses affect the traction performance of t-CVT significantly. Therefore, decreasing the spin losses can improve the reliability and fatigue life $^{[13]}$, increase the power density and torque capacity ${ }^{[7,14]}$, and greatly improve the traction efficiency of traction CVT $^{[15-17]}$.

Therefore, how to eliminate spin losses in traction CVT has become a hot research topic in this field. Typically, a kind of Shaft Drive CVT(SD-CVT) was proposed by Narita et al. ${ }^{[18]}$, where the spin was greatly decreased and the efficiency of SD-CVT was improved. Then, a novel double roller Full-Toroidal CVT(FT-CVT) was designed by Novellis et al. ${ }^{[17]}$, and this type of CVT can also significantly decrease the spin losses. However, the most representative research was the zero-spin design methodology developed by Li et al. ${ }^{[15,16]}$. The core idea of this methodology was that the original working surface of drive or driven components were redesigned to satisfy the zero-spin condition mentioned above. According to this method, the Logarithmic CVT(L-CVT) was firstly obtained by redesigning the original working surface of toroidal disk in HT-CVT (Li et al., 2015a; Li et al., 2015b). Sequentially, the integral CVT and Lambert CVT were obtained through the zero-spin design of FU-type CVT and tapered-roller and ring-disk CVT(TRCVT) ( $\mathrm{Li}$ et al., 2017). Recently, a kind of conical CVT was also acquired by redesign the roller disk type of CVT according to the zero-spin methodology, then the Swing Conical CVT (SRC-CVT) and Rolling Conical CVT (RC-CVT) were proposed, and the kinematics and efficiency of SRC-CVT was studied ${ }^{[19]}$.

In this paper, the study of design principles and traction performance of RC-CVT is conducted. As for RC-CVT, the conical disks are used as intermediate transmission elements, the rollers are used as input and output elements. To ensure zero-spin transmission characteristic, the limiting mechanism is used to restrict the vertex of conical disks on the fixed-point. At first, the specific structure and working principle of RC-CVT are described in detail. Afterwards, the kinematic qualities, including creep and spin, and contact models of RC-CVT are developed, then the efficiency computational model is carried out. Based on these computational models and parameters, the traction efficiency of RC-CVT are calculated. The results show that the RC-CVT can eliminate the spin loss theoretically, and the efficiency on fixed transmission ratio is consequently higher than that of HT-CVT.

\section{Geometric description}

As shown in Figure 2, this novel RC-CVT is composed of the input roller, the power transmitting conical disks and the output roller. The loading cam is a mechanism that utilizes balls constrained in wedges to create axial load, similar to most $\mathrm{HT}^{-\mathrm{CVT}^{[9]}}{ }^{\text {. To ensure zero-spin }}$ transmission characteristic, the limiting mechanism is used to restrict the vertexes of conical disks on the fixed-point $\mathrm{O}$. For this RC-CVT, the working principle is that the conical disks are driven to rotate around point $\mathrm{O}$, as shown with the hollow arrow, then the input roller and output roller can be consequently driven to adaptively move along the direction of solid arrow by the loading cam mechanism, thus the transmission ratio is changed.

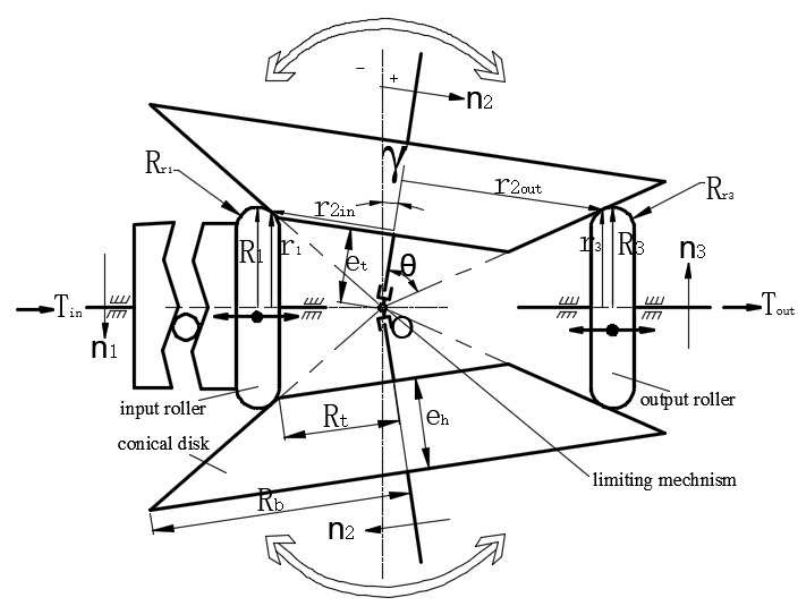

Figure 2 Geometrics of RC-CVT

To simplify the computation model, the geometric radii of output roller and input roller are set the same as $r_{0}$, which also means the cavity radius of RC-CVT.

$$
R_{1}=R_{3}=r_{0}
$$

According to the geometry, the rolling radius of input roller can be represented as: 


$$
r_{1}=R_{1}-R_{r 1}(1-\sin (\theta-\gamma))
$$

Similarly, the output roller as:

$$
r_{3}=R_{3}-R_{r 3}(1-\sin (\theta+\gamma))
$$

The rolling radii of conical disk at the input and output contacts are:

$$
\begin{aligned}
& r_{2_{\text {in }}}=\frac{\sin \theta}{\cos (\theta-\gamma)} r_{1} \\
& r_{2_{\text {out }}}=\frac{\sin \theta}{\cos (\theta+\gamma)} r_{3}
\end{aligned}
$$

Substituting equation (2) to (4), the equation (4) follows,

$$
r_{2_{\text {in }}}=\frac{\sin \theta}{\cos (\theta-\gamma)}\left[R_{1}-R_{r 1}(1-\sin (\theta-\gamma))\right]
$$

Similarly,

$$
r_{2_{\text {out }}}=\frac{\sin \theta}{\cos (\theta+\gamma)}\left[R_{3}-R_{r 3}(1-\sin (\theta+\gamma))\right](7)
$$

From the figure 2, the following relationships can also be acquired:

$$
\tan \theta=\frac{R_{t}}{e_{t}}=\frac{R_{b}}{e_{t}+e_{h}}
$$

Define structural parameter $k$ as the ratio of the top part height of conical disk $e_{t}$ and the cavity radius $r_{0}$. Hence, $k$ can be calculated by:

$$
k=\frac{e_{t}}{r_{0}}
$$

The theoretical transmission ratio is an important geometric parameter, which is defined as the ratio of input velocity and output velocity. For RC-CVT, it can be expressed as follows:

$$
i=\frac{\omega_{1}}{\omega_{3}}=\frac{\sin \theta}{\cos (\theta-\gamma)} \cdot \frac{\cos (\theta+\gamma)}{\sin \theta}=\frac{\cos (\theta+\gamma)}{\cos (\theta-\gamma)}
$$

\section{Creep and spin}

The practical transmission ratio is different from the theoretical transmission ratio because of creep, which is the velocity difference on the contacting surfaces in the primary direction of traction. Generally, the creep is inevitable for producing the tangential force for traction, which can be represented by the creep coefficient. For input contact and output contacts, the creep coefficients are respectively defined as:

$$
\begin{aligned}
& C_{r_{\text {in }}}=\frac{V_{1}-V_{2}}{V_{1}}=\frac{\left|\omega_{1}\right| r_{1}-\left|\omega_{2}\right| r_{2} \text { in }}{\left|\omega_{1}\right| r_{1}} \\
& C_{r_{\text {out }}}=\frac{V_{2}-V_{3}}{V_{2}}=\frac{\left|\omega_{2}\right| R_{23}-\left|\omega_{3}\right| r_{3}}{\left|\omega_{2}\right| r_{2} \text { out }}
\end{aligned}
$$

Hence, the $\frac{\left|\omega_{2}\right|}{\left|\omega_{1}\right|}$ can be derived from the equation (11), it follows,

$$
\frac{\left|\omega_{2}\right|}{\left|\omega_{1}\right|}=\left(1-C_{r_{i n}}\right) \frac{r_{1}}{r_{2_{\text {in }}}}=\left(1-C_{r_{i n}}\right) \frac{\cos (\theta-\gamma)}{\sin \theta}
$$

Similarly, the $\frac{\left|\omega_{3}\right|}{\left|\omega_{2}\right|}$ follows,

$$
\frac{\left|\omega_{3}\right|}{\left|\omega_{2}\right|}=\left(1-C_{r_{\text {out }}}\right) \frac{\sin \theta}{\cos (\theta+\gamma)}
$$

Therefore, the practical speed ratio $s_{r}$ can be defined and represented as:

$$
s_{r}=\frac{\left|\omega_{3}\right|}{\left|\omega_{1}\right|}=\left(1-C_{r_{\text {in }}}\right)\left(1-C_{r_{\text {out }}}\right) \frac{\cos (\theta-\gamma)}{\cos (\theta+\gamma)}
$$

Further, the spin velocities of input point and output contact are described as follows:

$$
\begin{aligned}
& \omega_{21 \text { spin }}=\left|\omega_{1}\right| \cos (\theta-\gamma)-\left|\omega_{2}\right| \sin \theta \\
& \omega_{23 \text { spin }}=\left|\omega_{3}\right| \cos (\theta+\gamma)-\left|\omega_{2}\right| \sin \theta
\end{aligned}
$$

At the same time, the corresponding spin ratios are defined as: $\sigma_{21}=\frac{\omega_{21 \text { spin }}}{\left|\omega_{1}\right|}$ and $\sigma_{23}=\frac{\omega_{23 \text { spin }}}{\left|\omega_{3}\right|}$.

Therefore,

$$
\begin{aligned}
& \sigma_{21}=\cos (\theta-\gamma)-\frac{\left|\omega_{2}\right|}{\left|\omega_{1}\right|} \sin \theta \\
& \sigma_{23}=\frac{\left|\omega_{2}\right|}{\left|\omega_{3}\right|} \sin \theta-\cos (\theta+\gamma)
\end{aligned}
$$

Substituting the (13) and (18),

$$
\begin{gathered}
\sigma_{21}=C_{r_{\text {in }}} \cos (\theta-\gamma) \\
\sigma_{23}=-\frac{C_{r_{\text {out }}}}{\left(1-C_{r_{\text {out }}}\right)} \cos (\theta+\gamma)
\end{gathered}
$$

From the above equations (20) and (21), it is obvious that the spin ratios are zero when creep coefficients are not considered. So, the RC-CVT can eliminate the spin losses theoretically. But the practical spin ratio is not zero because of non-zero creep during the power transmitting. 


\section{Contact ellipse}

According to the Hertz contact theory, when there is a normal loading force between a roller and a conical disk, the contact point should be an ellipse. The direction and dimension of the ellipse are related to the normal force of the contact point, the material properties and structural dimensions of contacted components.

For RC-CVT, the most critical problems for contact model is to determine the principal curvature radii of the direction of traction and side slip.

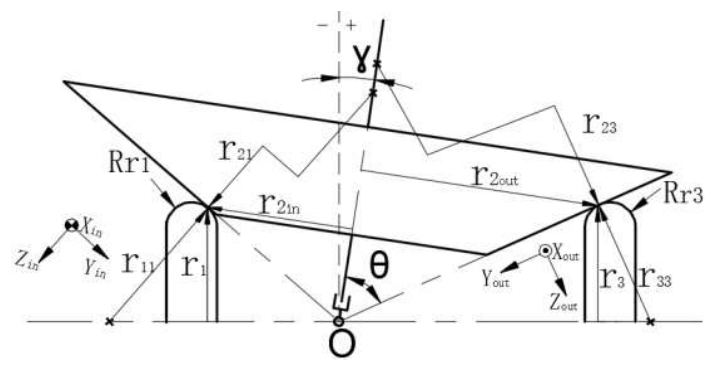

Figure 3 The reference frames and principal curvature radii for the rollers and conical disks

The contact between the roller and conical disks, and the reference frames are shown in figure 3. The equivalent curvature radii of curvature along the $\mathrm{x}$ direction (traction direction) and y direction (side slip direction) are:

$$
\begin{aligned}
& \frac{1}{\rho_{e q X}}=\frac{1}{\mathrm{r}_{a_{x}}}+\frac{1}{\mathrm{r}_{b_{x}}} \\
& \frac{1}{\rho_{e q Y}}=\frac{1}{\mathrm{r}_{a_{y}}}+\frac{1}{\mathrm{r}_{b_{y}}}
\end{aligned}
$$

where the subscript a refers to the roller and $\mathrm{b}$ to conical disk.

Then the equivalent radius of curvature is defined as

$$
\frac{1}{\rho_{e q}}=\frac{1}{\rho_{e q X}}+\frac{1}{\rho_{e q Y}}
$$

For the input roller, $r_{a_{x i n}}$ and $r_{a_{y i n}}$ can be represented as,

$$
\begin{gathered}
\mathrm{r}_{a_{x i n}}=\mathrm{r}_{11}=\frac{r_{1}}{\sin (\theta-\gamma)}=\frac{R_{1}-R_{r 1}(1-\sin (\theta-\gamma))}{\sin (\theta-\gamma)} \\
\mathrm{r}_{a_{y i n}}=R_{r 1}
\end{gathered}
$$

For the conical disk, the $r_{b_{x i n}}$ and $r_{b_{y i n}}$ are determined as:

$$
\begin{gathered}
\mathrm{r}_{b_{x i n}}=\mathrm{r}_{21}=\frac{r_{2_{\text {in }}}}{\cos \theta}=\frac{\left(R_{1}-R_{r 1}(1-\sin (\theta-\gamma))\right) \tan \theta}{\cos (\theta-\gamma)} \\
\mathrm{r}_{b_{\text {yin }}}=\infty
\end{gathered}
$$

Then, the two equivalent curvature radii at the input contact along the $\mathrm{x}$ and $\mathrm{y}$ directions can be determined respectively:

$$
\begin{gathered}
\frac{1}{\rho_{\text {eqXin }}}=\frac{1}{\mathrm{r}_{a_{x i n}}}+\frac{1}{\mathrm{r}_{b_{x i n}}}=\frac{\sin (\theta-\gamma) \tan \theta+\cos (\theta-\gamma)}{\left(R_{1}-R_{r 1}(1-\sin (\theta-\gamma))\right) \tan \theta} \\
\frac{1}{\rho_{\text {eqYin }}}=\frac{1}{\mathrm{r}_{a_{y i n}}}+\frac{1}{\mathrm{r}_{b_{y i n}}}=\frac{1}{R_{r 1}}
\end{gathered}
$$

Similarly, the two principal curvature radii of output roller and conical disk at output contact can be determined as follows:

$$
\begin{aligned}
\mathrm{r}_{a_{\text {xout }}=}=\mathrm{r}_{33}= & \frac{r_{3}}{\sin (\theta+\gamma)}=\frac{R_{3}-R_{r 3}(1-\sin (\theta+\gamma))}{\sin (\theta+\gamma)} \\
& \mathrm{r}_{a_{\text {yout }}}=R_{r 3} \\
\mathrm{r}_{b_{\text {xout }}}=\mathrm{r}_{23}= & \frac{r_{2_{\text {out }}}}{\cos \theta}=\frac{\left(R_{3}-R_{r 3}(1-\sin (\theta+\gamma))\right) \tan \theta}{\cos (\theta+\gamma)} \\
& \mathrm{r}_{b_{\text {yout }}}=\infty
\end{aligned}
$$

Therefore, the equivalent curvature radii at the output contact along the $\mathrm{x}$ and $\mathrm{y}$ directions can be determined respectively:

$$
\begin{gathered}
\frac{1}{\rho_{\text {eqXout }}}=\frac{1}{\mathrm{r}_{a_{\text {xout }}}}+\frac{1}{\mathrm{r}_{b_{\text {xout }}}}=\frac{\sin (\theta+\gamma) \tan \theta+\cos (\theta+\gamma)}{\left(R_{3}-R_{r 3}(1-\sin (\theta+\gamma))\right) \tan \theta} \\
\frac{1}{\rho_{\text {eqYout }}}=\frac{1}{\mathrm{r}_{a_{\text {yout }}}}+\frac{1}{\mathrm{r}_{b_{\text {yout }}}}=\frac{1}{R_{r 3}}
\end{gathered}
$$

On this basis, the equivalent radii of curvature at input and output contact are then obtained,

$$
\begin{gathered}
\frac{1}{\rho_{\text {eqin }}}=\frac{\sin (\theta-\gamma) \tan \theta+\cos (\theta-\gamma)}{\left(R_{1}-R_{r 1}(1-\sin (\theta-\gamma))\right) \tan \theta}+\frac{1}{R_{r 1}} \\
\frac{1}{\rho_{\text {eqout }}}=\frac{\sin (\theta+\gamma) \tan \theta+\cos (\theta+\gamma)}{\left(R_{3}-R_{r 3}(1-\sin (\theta+\gamma))\right) \tan \theta}+\frac{1}{R_{r 3}}
\end{gathered}
$$

It is useful to define the dimensionless form of these equivalent radii of curvature as $\tilde{\rho}_{e q}=\rho_{e q} / r_{0}$, which has also described detailly in Carbone's article (2004).

According to the Hertz contact theory, the contact stress in the contact area can be expressed as follows:

$$
\mathrm{p}=\mathrm{p}_{\max }\left(1-\frac{x^{2}}{b^{2}}-\frac{y^{2}}{a^{2}}\right)
$$

The maximum Hertz contact stress $\mathrm{p}_{\max }$ is:

$$
\mathrm{p}_{\max }=\frac{3 \mathrm{~F}_{N}}{2 \pi a b}
$$

where $\mathrm{a}$ is the long axis of the contact ellipse, and $\mathrm{b}$ is the short axis, and $\mathrm{F}_{N}$ is the normal force at contact point.

In the researches of elliptical contact at isothermal EHL state by Hamrock and Dowson ${ }^{[20,21]}$ the iterative algorithm 
proposed by Harris ${ }^{[22]}$ was used to determine the parameter $\mathrm{a}$ and $\mathrm{b}$ with higher precision, as follows:

$$
\begin{gathered}
\mathrm{a}=\left(\frac{6 f^{2} \varepsilon \rho_{e q} \mathrm{~F}_{N}}{\pi E^{\prime}}\right)^{1 / 3} \\
\mathrm{~b}=\frac{a}{f}
\end{gathered}
$$

where $\rho_{e q}$ is the equivalent curvature radius, it can be determined by the $\rho_{\text {eqXin }}$ and $\rho_{\text {eqYin }}$, or, $\rho_{\text {eqXout }}$ and $\rho_{\text {eqYout }}$.

While $E^{\prime}$ is the equivalent elastic modulus, it can be determined by the following equation:

$$
\frac{2}{E^{\prime}}=\frac{1-v_{1}^{2}}{E_{1}}+\frac{1-v_{2}^{2}}{E_{2}}
$$

The parameters $f$ and $\varepsilon$ can be determined as following:

$$
\begin{aligned}
& f=1.0339\left(\frac{\rho_{e q Y}}{\rho_{e q X}}\right)^{0.636} \\
& \varepsilon=1.0003+\frac{0.5968}{\frac{\rho_{e q Y}}{\rho_{e q X}}}
\end{aligned}
$$

According to the method by Carbone ${ }^{[23]}$, we define a characteristic length,

$$
\Lambda=\sqrt[3]{\frac{6 F_{N} r_{0}}{\pi E^{\prime}}}
$$

The dimensionless semi-axis of contact ellipse can be described as,

$$
\begin{aligned}
& \tilde{a}_{X}=\frac{\mathrm{a}}{\Lambda}=\sqrt[3]{f^{2} \varepsilon\left(\rho_{e q} / \mathrm{r}_{0}\right)} \\
& \tilde{a}_{Y}=\frac{\mathrm{b}}{\Lambda}=\frac{\tilde{a}_{X}}{f}=\sqrt[3]{\frac{\varepsilon\left(\rho_{e q} / \mathrm{r}_{0}\right)}{f}}
\end{aligned}
$$

Similarly, the $\mathrm{p}$ is then written in dimensionless form as,

$$
\tilde{\mathrm{p}}=\frac{\mathrm{p} \Lambda^{2}}{\mathrm{~F}_{N}}=\tilde{\mathrm{p}}_{\max } \sqrt{1-X^{2}-Y^{2}}
$$

where $\tilde{\mathrm{p}}_{\text {max }}=\frac{\mathrm{p}_{\max } \Lambda^{2}}{\mathrm{~F}_{N}}=\frac{3 \mathrm{~F}_{N}}{2 \pi \tilde{a}_{X} \tilde{a}_{Y}}, X=\frac{x}{\tilde{a}_{X}}$ and $Y=\frac{y}{\tilde{a}_{Y}}$.

\section{Design principles}

To compare with HT-CVT, the $r_{0}$ and $\theta$ are given as the same as the research by Carbone ${ }^{[23]}$. Then the conical disks and rollers can be compactly designed through the following steps.

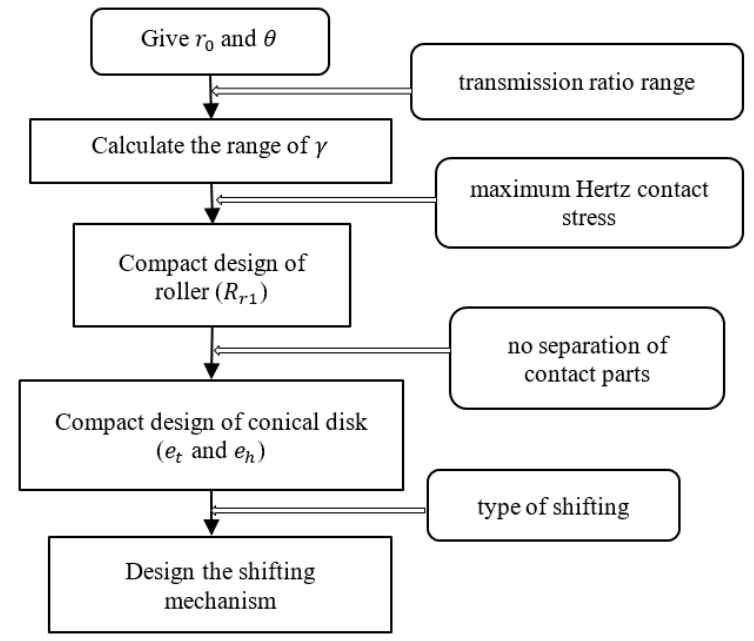

Figure 4 The design flowchart of RC-CVT

In this paper, the following relationship is assumed to simplify the design process:

$$
R_{r 1}=R_{r 3}=d r_{0}
$$

Step 1: The given parameters are $r_{0}=40 \mathrm{~mm}, \theta=$ $\pi / 3$, and the transmission ratio range is $0.5 \sim 2$. According to the equation (10), the range of $\gamma$ can be obtained, which is $-0.19 \mathrm{rad}-0.19 \mathrm{rad}$.

Substituting the equation (50) to (2), it follows,

$$
r_{1}=(1-d+d \sin (\theta-\gamma)) r_{0}
$$

For RC-CVT, the $r_{1}>0$ and $r_{2_{\text {in }}}>0$ should be ensured. According to the equations (4) and (51), then we can get $0<d<4$. This is a restriction of the next step.

Step 2: According to the Hertz contact theory described in section 4 , the relationship of $\mathrm{i}$ and $\mathrm{p}_{\max }$ at the situation of different $R_{r 1}$ can be acquired, as shown in figure 5 . It is found that $\mathrm{d}=2$ is a better choice to make the maximum Hertz contact stress $\mathrm{p}_{\max }$ at a relatively small level, which is less than $3 \mathrm{Gpa}$, similar to HT-CVT.

Then, the roller can be designed, as shown in the following figure 6 , where the theoretical working curve of roller is $\widehat{O_{1} A}$ and $\widehat{O_{2} A}$. So, we can determine one segment from this curve to design the final roller. For example, $O_{1} P_{1} O_{2} P_{2}$ forms a roller, and the $A B$. represents the rotation axis. 


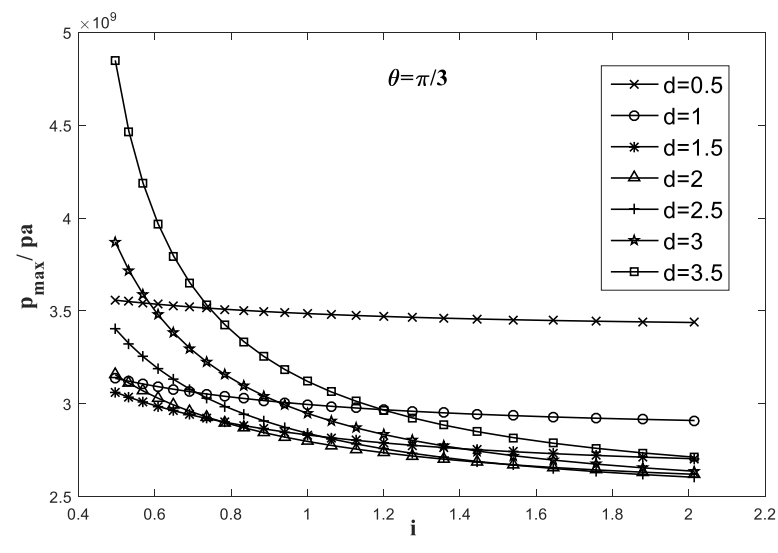

Figure 5 The design flowchart of RC-CVT

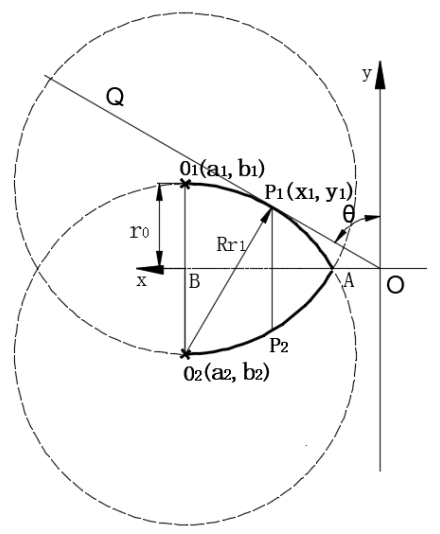

Figure 6 The designed curve of roller for RC-CVT

Since the half cone angle is $\pi / 3$, the theoretical working curve of conical disk can be assumed as the line $O Q$. So, the point $O$ is the intersection of $O Q$ and $A B$, and $O Q$ and $\widehat{O_{1} A}$ are tangent to point $P_{1}\left(x_{1}, y_{1}\right)$. Therefore, a coordinate system can be established as in figure6. This shows the contact situation of roller and conical disk when $\gamma=0$. Then the coordinates of point $P_{1}, O_{1}, B, O_{2}$ and $P_{2}$ can be obtained, which are $P_{1}\left((3-\sqrt{3}) r_{0},(\sqrt{3}-1) r_{0}\right), O_{1}\left((4-\sqrt{3}) r_{0}, r_{0}\right), B((4-$ $\left.\sqrt{3}) r_{0}, 0\right) \quad, \quad O_{2}\left((4-\sqrt{3}) r_{0},-r_{0}\right) \quad$ and $\quad P_{2}((3-$ $\left.\sqrt{3}) r_{0},-(\sqrt{3}-1) r_{0}\right)$. To design a compact roller, the inversion method is used to determine the extreme contact position of roller when the conical disk is shifted from $-0.19 \mathrm{rad}$ to $0.19 \mathrm{rad}$, as shown in figure 7 .

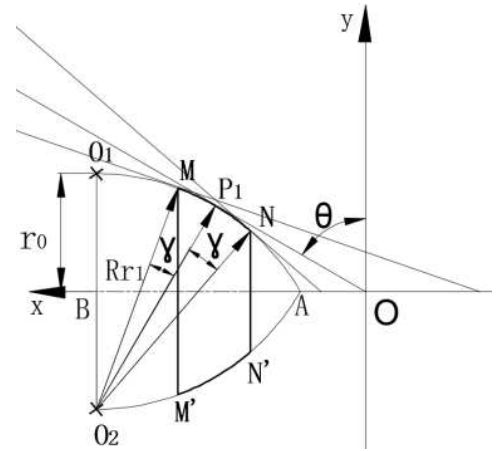

Figure 7 The compact design of roller for RC-CVT

Step 3: When the conical disk is tilted to extreme position, that means the tilting angle of conical disk has reached its maximum $\gamma_{\max }$, the no separation relationships of rollers and conical disk should be satisfies.

Based on the relationship in geometric description, the following can be acquired,

$$
\begin{aligned}
& R_{t} \leq \frac{\sin \theta}{\cos \left(\theta-\gamma_{\max }\right)}\left[r_{0}-r_{0}\left(1-\sin \left(\theta-\gamma_{\max }\right)\right)\right] \\
& R_{b} \geq \frac{\sin \theta}{\cos \left(\theta+\gamma_{\max }\right)}\left[r_{0}-r_{0}\left(1-\sin \left(\theta+\gamma_{\max }\right)\right)\right]
\end{aligned}
$$

Hence, $R_{t} \leq 27.0976 \mathrm{~mm}, R_{b} \geq 94.1271 \mathrm{~mm}$ can be acquired. To design a compact conical disk, the $R_{t}$ should take the maximum value and $R_{b}$ takes the minimum. Considering a safety margin, the $R_{t}$ and $R_{b}$ can be determined. Then, the $e_{t}$ and $e_{h}$ can be obtained based on equation (8).

Step 4: For RC-CVT, the speed changing motion of conical disks is rotation around point $\mathrm{O}$, which can be realized by lots of mechanisms actually. In this paper, a simple shifting mechanism ${ }^{[24]}$ is used, where the shifting gears can be driven by a couple of synchronous motors.

Finally, the RC-CVT can be designed. However, this design principle just provides a simple and general method, which may be further refined when conduct a specific design of variable RC-CVTs.

\section{Traction efficiency}

In terms of efficiency calculation, Carbone ${ }^{[23]}$ has given a more accurate computational method, which has been widely used especially in the efficiency calculation of toroidal CVT ${ }^{[15-17,25,26]}$. In this method, the thickness of the oil film is explained with Hamrock and Dowson formulas ${ }^{[27]}$, the oil temperature is assumed at the flash point and pressure distribution in the contact area obeys the Hertz contact theory. The rheological behavior of traction fluid is described using the Bair and Winer Non-Newtonian 
model ${ }^{[28]}$, and the influence of the pressure on the fluid viscosity is computed with Roelands model ${ }^{[28]}$, which is more suitable for the situation of high pressure in the contact area. This computational method is also developed in this paper to calculate the efficiency of RC-CVT.

The traction efficiency of RC-CVT is defined as follows:

$$
v=\left(1-C_{r}\right) \frac{\mu_{o u t}-\chi_{o u t} \cos (\theta+\gamma)}{\mu_{\text {in }}+\chi_{\text {in }} \cos (\theta-\gamma)}
$$

where $1-C_{r}=\left(1-C_{r_{\text {in }}}\right)\left(1-C_{r_{\text {out }}}\right), \quad C_{r_{\text {in }}}$ and $C_{r_{\text {out }}}$ are the input and output creep coefficients. Additionally, $\mu_{\text {in }}$ and $\mu_{\text {out }}$ are the input and output traction coefficients, $\chi_{\text {in }}$ and $\chi_{\text {out }}$ are the spin momentum coefficients, these four parameters can be calculated by the numerical integration in the region of contact area, which are all described in detail by Carbone ${ }^{[23]}$.

According to the aforementioned calculation models, the traction performance is analysed, where the geometrical quantities are reported in Table 2. To compare with HT-CVT, the geometrical quantities and fluid properties are set as similar as that of HT-CVT by Carbone ${ }^{[23]}$. As developed in section 5, the maximum Hertz contact stress $\mathrm{p}_{\text {max }}$ has been optimized to less than $3 \mathrm{Gpa}$ by compactly designing the roller. Then the Hertz contact stress of RC-CVT is similar to HT-CVT, which is also the equality condition to conduct the comparation.

For the designed RC-CVT, the parameter $k$ reflects the structural compactness. In fact, the $k$ also influences the maximal tilting angle of conical disk $\gamma_{\max }$, which further determines the transmission ratio range. According to the compactly designed roller and conical disk, the structural parameter $k$ can be determined as 0.3175 .

Table 2 Geometric data of RC-CVT

\begin{tabular}{ll}
\hline RC-CVT & \\
\hline Cavity radius & $r_{0}=40 \mathrm{~mm}$ \\
Half cone-angle & $\theta=\pi / 3$ \\
Transmission ratio range & $\mathrm{i}=0.5 \sim 2$ \\
Input angular velocity & $\left|\omega_{1}\right|=2000 \mathrm{rpm}$ \\
Number of the cavity & $\mathrm{m}=1$ \\
Number of conical disks & $\mathrm{n}=2$ \\
Young's Modulus $\left(\mathrm{E}_{1}\right.$ and $\left.\mathrm{E}_{2}\right)$ & $2.1 \times 10^{11} \mathrm{~N} / \mathrm{m}^{2}$ \\
Poisson ratios $\left(v_{1}\right.$ and $\left.v_{2}\right)$ & 0.3 \\
\hline
\end{tabular}

Subsequently, the practical spin ratio of RC-CVT is calculated and compared with HT-CVT, the results are shown in Figure 8. It can be seen that the practical spin ratio of RC-CVT is not zero when creep coefficients are considered, but the value is far less than that of HT-CVT, which is close to zero. Therefore, the RC-CVT is theoretically eliminate the spin losses.

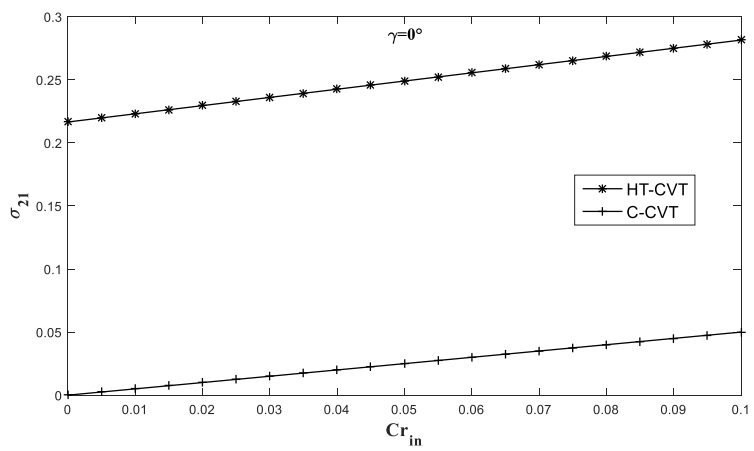

(a)

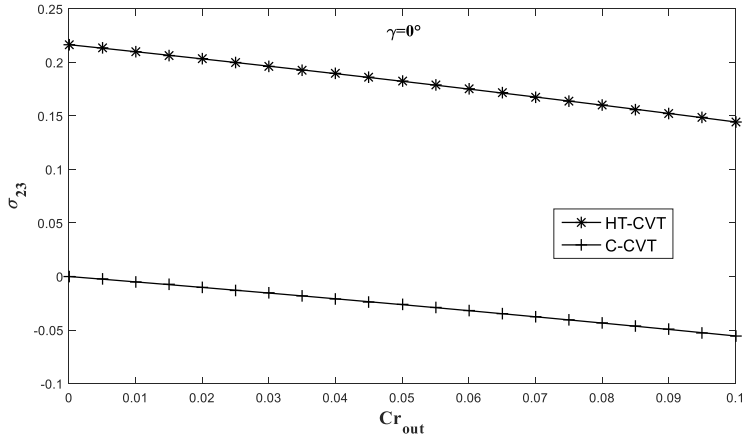

(b)

Figure 8 The practical spin ratio comparation between HT-CVT and RC-CVT: (a) The practical spin ratio at input contact; (b) The practical spin ratio at output contact

From the equations (61), it is obvious that the spin momentum coefficients $\chi_{i n}$ is mainly determined by the spin moment $M_{S_{i n}}$. The input torque coefficient $t_{i n}$ is the dimensionless form of $T_{i n}$, which represents the input torque. The relationship between the input spin momentum coefficients $\chi_{i n}$ and the input torque coefficient $t_{i n}$ of the HT-CVT and RC-CVT are shown in Figure 9. From this figure, it is easy to find that the spin moment $\chi_{\text {in }}$ of the RC-CVT is greatly reduced compared with the HT-CVT. But the fact is that the practical $\chi_{i n}$ are not zero, because of the larger $\mathrm{t}_{i n}$, the greater $C_{r_{i n}}$ (see in figure 15), which could cause the additional spin losses. Additionally, the situation of output contact is similar to this of input contact. 


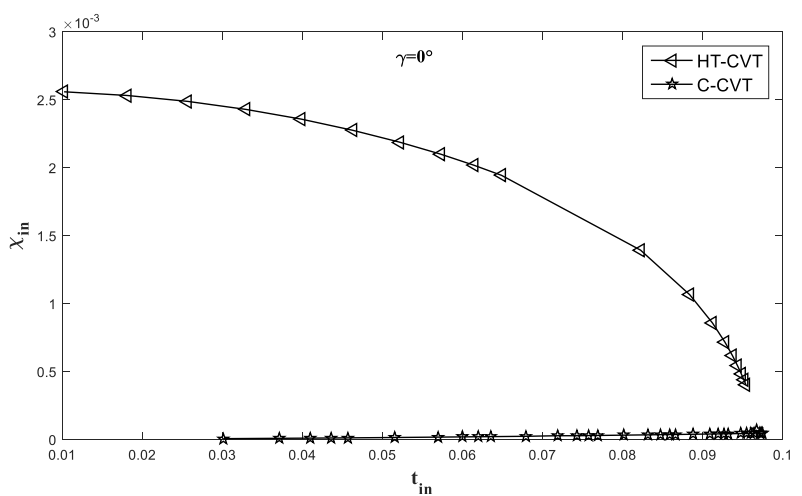

Figure 9 The spin moment $\chi_{i n}$ as a function of input traction coefficient $t_{\text {in }}$

Moreover, the efficiency of the RC-CVT and HT-CVT as a function of the input torque coefficient $t_{\text {in }}$ are shown in Figure 10. It is apparent that the efficiency of the RC-CVT is higher than that of the HT-CVT, especially when the input torque coefficient $t_{i n}$ is at the smaller stage. This is indeed the advantage of eliminating spin losses. It is worth pointed out that the efficiency rapidly drops as $t_{i n}$ approaches the limited value, because the creep coefficient fast increase at this situation (see in figure 15).

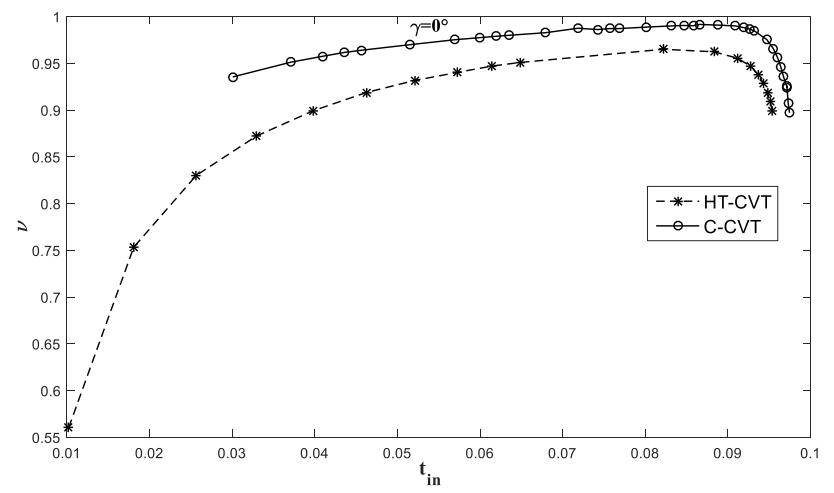

Figure 10 The efficiency of the CVTs as a function of the input traction coefficient $t_{\text {in }}$

The figure 11 gives the efficiency $v$ as a function of the overall creep coefficient $C_{r}$. Compare with HT-CVT, the efficiency of RC-CVT is much higher than that of HT-CVT when $C_{r}$ is smaller than 0.03 , the reason is that the traction losses are dominated by spin at this situation, but the spin of RC-CVT is eliminated theoretically. However, as $C_{r}$ further increases, the creep losses become the dominator, which is considered much greater than spin, the traction efficiency therefore linearly drops to the similar level of these two CVTs. It should be pointed out that the same input torque coefficient $t_{i n}$ would cause the different creep for this two CVTs. In fact, the creep coefficient $C_{r}$ of HT-CVT is more sensitive to $\mathrm{t}_{i n}$, which means a small input torque of HT-CVT may cause a relatively larger creep compared with RC-CVT. In particular, as shown in figure 11 , when $t_{i n}$ increases at a smaller stage, the $C_{r}$ of HT-CVT increases obviously faster than RC-CVT. Only when the $t_{\text {in }}$ increases to greater than 0.085 , the $C_{r}$ of RC-CVT increases rapidly, and the HT-CVT has a similar feature.

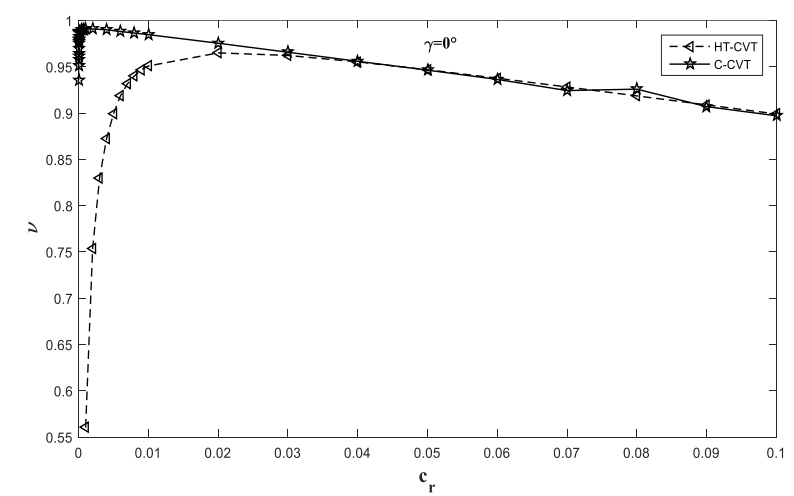

Figure 11 The efficiency of the CVTs as a function of the creep coefficient $C_{r}$

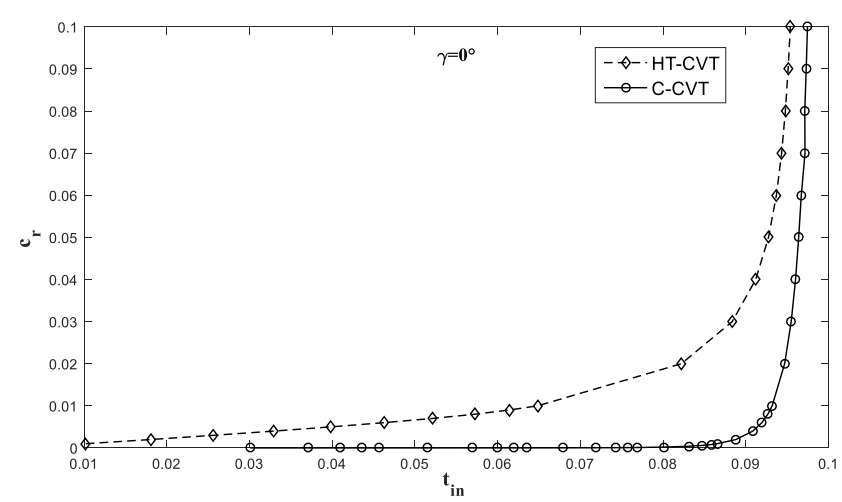

Figure 12 The relationship between $\mathrm{t}_{i n}$ and $C_{r}$ of HT-CVT and RC-CVT

Totally, the RC-CVT has a higher traction efficiency on fixed transmission ratio than that of HT-CVT, especially when $\mathrm{t}_{\text {in }}$ or $C_{r}$ is relatively small. Therefore, the $\mathrm{RC}-\mathrm{CVT}$ is considered to have the potential to be a new generation of traction CVT with higher efficiency.

\section{Conclusions}

In this paper, the design principles and traction performance are studied. The following conclusions can be drawn:

(1) The kinematics and contact model of RC-CVT are studied, a design flowchart of RC-CVT is given. By the 
proposed design principles, the roller and conical disk, as well as the shifting mechanism are designed. Especially, the edge radius of roller $R_{r}$ significantly influences the maximum Hertz contact stress, and the recommended $R_{r}$ of designed RC-CVT is $R_{r}=2 r_{0}$.

(2) The RC-CVT just theoretically eliminates the spin losses. When the creep coefficients are considered, the practical spin ratio of RC-CVT is not zero. However, the spin moment is far less than that of HT-CVT, reduced by more than $90 \%$ at the input contact.

(3) The RC-CVT has been proved to have relatively higher traction efficiency on fixed transmission ratio than that of HT-CVT, especially when the $\mathrm{t}_{i n}$ and $C_{r}$ are at a small stage.

Although the traction efficiency performance of RC-CVT is studied, it is just the efficiency calculation on fixed transmission ratio without considering the shifting loss. Therefore, the overall efficiency including the shifting loss of the dynamic speed changing process and the experimental verification are the next important research work. In addition, the influence of different parameters to traction efficiency has not been discussed completely, which also deserves to be studied in the future.

\section{Declaration}

\section{Funding}

Supported by the National Natural Science Foundation of China Youth Science Foundation[Grant No.51905447], and Starting Fund for Teacher Development and Research of Chengdu University of Technology (Grant numbers 10912-KYQD2019_07733)

\section{Availability of data and materials}

The datasets supporting the conclusions of this article are included within the article.

\section{Authors' contributions}

The author' contributions are as follows: Chao Li wrote the manuscript and in charge of the main computation model; Xiu-quan Cao assisted with calculation and figures; Qing-tao Li modified the full text logic and guided the analysis of the results.

\section{Competing interests}

The authors declare no competing financial interests.

\section{Consent for publication}

Not applicable

\section{Ethics approval and consent to participate}

Not applicable

\section{References}

[1] Park N G, Ryu J H, Lee H W, et al. Development of the Inner Spherical CVT for a motorcycle[J]. International Journal of Automotive Technology. 2009, 10(3): 341-346.

[2] Shen C J, Yuan S H, Wei C, et al. Transmission efficiency of hydraulic clamp type traction transmission device[J]. Journal of Xi'an Jiaotong University. 2012, 46(7): 32-37.

[3] Fuchs R, Hasuda Y, Rothernbuehler Y, et al. Control Concepts of Continuously Variable Transmissions (CVT)[J]. JTEKT Engineering Journal English Edition. 2006(1001E): 24-29.

[4] Ryu W, Kim H. CVT ratio control with consideration of CVT system loss[J]. International Journal of Automotive Technology. 2008, 9(4): 459-465.

[5] Carbone G, Mangialardi L, Bonsen B, et al. CVT dynamics: Theory and experiments[J]. Mechanism and Machine Theory. 2007, 42(4): 409-428.

[6] Sanda S, Hayakawa K. Traction Drive System and its Characteristics as Power Transmission[J]. R\&D Review of Toyota CRDL. 2005, 40(3): 30-39.

[7] Itagaki $\mathrm{H}$, Hashiguchi $\mathrm{H}$, Kita $\mathrm{M}$, et al. Development of a High-Power Two-Roller Traction Tester and Measurement of Traction Curves[J]. Tribology Online. 2016, 11(6): 661-674.

[8] Li X M, Guo F, Fan B, et al. Influence of spinning on the rolling EHL films[J]. Tribology International. 2010, 43(11): 2020-2028.

[9] Zhang Y, Zhang X, Tobler W. A Systematic Model for the Analysis of Contact, Side Slip and Traction of Toroidal Drives[J]. Journal of Mechanical Design. 2000, 122(4): 523-528.

[10] Cretu O S G R P. Traction Drive With Reduced Spin Losses[J]. Journal of tribology. 2003, 3(125): 507-512.

[11] Newall J, Lee A. Measurement and prediction of spin losses in the EHL point contacts of the full toroidal variator[J]. Tribology Series. 2003, 43: 769-779.

[12] Kim J, Choi K H. Spin loss analysis of friction drives: spherical and semi-spherical CVT[J]. International Journal of Automotive Technology. 2003, 4(4): 165-172.

[13] Yan X, Wang X, Zhang Y. A numerical study of fatigue life in non-Newtonian thermal EHL rolling-sliding contacts with spinning[J]. Tribology International. 2014, 80: 156-165.

[14] Yamanaka M, Narita Y, Igari G E A. Study of Shaft Drive Continuously Variable Transmission : 2nd Report, Effect of Low Spin Disk [C]. 2004.

[15] Li Q T, Wu J, Li H, et al. A Mathematical Method for Eliminating Spin Losses in Toroidal Traction Drives[J]. Mathematical Problems in Engineering. 2015, 2015: 1-10.

[16] $\mathrm{Li} \mathrm{Q} \mathrm{T,} \mathrm{Li} \mathrm{H,} \mathrm{Yu} \mathrm{D} \mathrm{P,} \mathrm{et} \mathrm{al.} \mathrm{A} \mathrm{novel} \mathrm{continuously} \mathrm{variable}$ transmission with logarithmic disc generatrix[J]. Mechanism and Machine Theory. 2015, 93: 147-162.

[17] De Novellis L, Carbone G, Mangialardi. Traction and Efficiency Performance of the Double Roller Full-Toroidal Variator: A Comparison with Half- and Full-Toroidal Drives[J]. Journal of Mechanical Design. 2012, 134(7): 71005.

[18] Narita Y, Yamanaka M, Inoue K. Optimum Design of Disk and Roller Shapes for Shaft Drive Continuously Variable Transmission[J]. Transactions of the Japan Society of Mechanical Engineers. 2006, 72(716): 1265-1270.

[19] $\mathrm{Li} \mathrm{C}$, $\mathrm{Li} \mathrm{H}, \mathrm{Li} \mathrm{Q}$, et al. Modeling, kinematics and traction 
performance of no-spin mechanism based on roller-disk type of traction drive continuously variable transmission[J]. Mechanism and Machine Theory. 2019, 133: 278-294.

[20] Hamrock B J, Dowson D. Isotermal Elastohydrodynamic Lubrication of Point Contacts: Part I - Theoretical Formulation[J]. Journal of Lubrication Technology, Trans. 1976, 98(3).

[21] Hamrock B J, Dowson D. Isothermal Elastohydrodynamic Lubrication of Point Contacts: part II -Ellipticity parameter results[J]. Journal of Lubrication Technology, Trans. 1976, 98(3).

[22] Harris T A. Rolling bearing analysis[M]. John Wiley \& Sons. Inc., 1966.

[23] Carbone G, Mangialardi L, Mantriota G. A comparison of the performances of full and half toroidal traction drives[J]. Mechanism and Machine Theory. 2004, 39(9): 921-942.

[24] Li C, Yao J, Li H, et al. Research on a novel configuration synthesis method of no-spin traction continuously variable transmission[J]. Mechanism and Machine Theory. 2020, 152: 103963.

[25] Delkhosh M, Foumani M S, Boroushaki M, et al. Geometrical optimization of half toroidal continuously variable transmission using particle swarm optimization[J]. Scientia Iranica. 2011, 18(5): 1126-1132.

[26] Delkhosh M, Foumani M S. Multi-objective geometrical optimization of full toroidal CVT[J]. International Journal of Automotive Technology. 2013, 14(5): 707-715.

[27] Dowson D. Modelling of Elastohydrodynamic Lubrication of Real Solids by Real Lubricants[J]. Meccanica. 1998, 33(1): 47-58.

[28] Hamrock B J. Fundamentals of fluid film lubrication[M]. McGraw-Hill, 1994: 59.

\section{Biographical notes}

Chao Li, born in 1989, is currently an associate research fellow at The College of Nuclear Technology and Automation Engineering, Chengdu University of Technology, China. He received his doctor degree on mechanical design and theory from Sichuan University, China, in 2019. His research interests include machine and mechanism theory, robotics and new traction continuously variable transmission technology.

Tel: +86-182-02822945; E-mail: lichao19@cdut.edu.cn

Xiu-quan Cao, born in 1989, is currently a lecturer at Sichuan University of Science and Engineering, China. He received his doctor degree on mechanical design and theory in Sichuan University, China, in 2017.

E-mail: cao_comeon@126.com

Qing-tao Li, born in 1987, is currently an associate professor at School of Mechanical Engineering, Xihua University, China. He received his doctor degree on mechanical design and theory from Sichuan University, China, in 2016. His research interests include hydro-mechainal transmission, machine and mechanism theory, and new traction continuously variable transmission technology.

E-mail: liqingtao@mail.xhu.edu.cn 
Figures

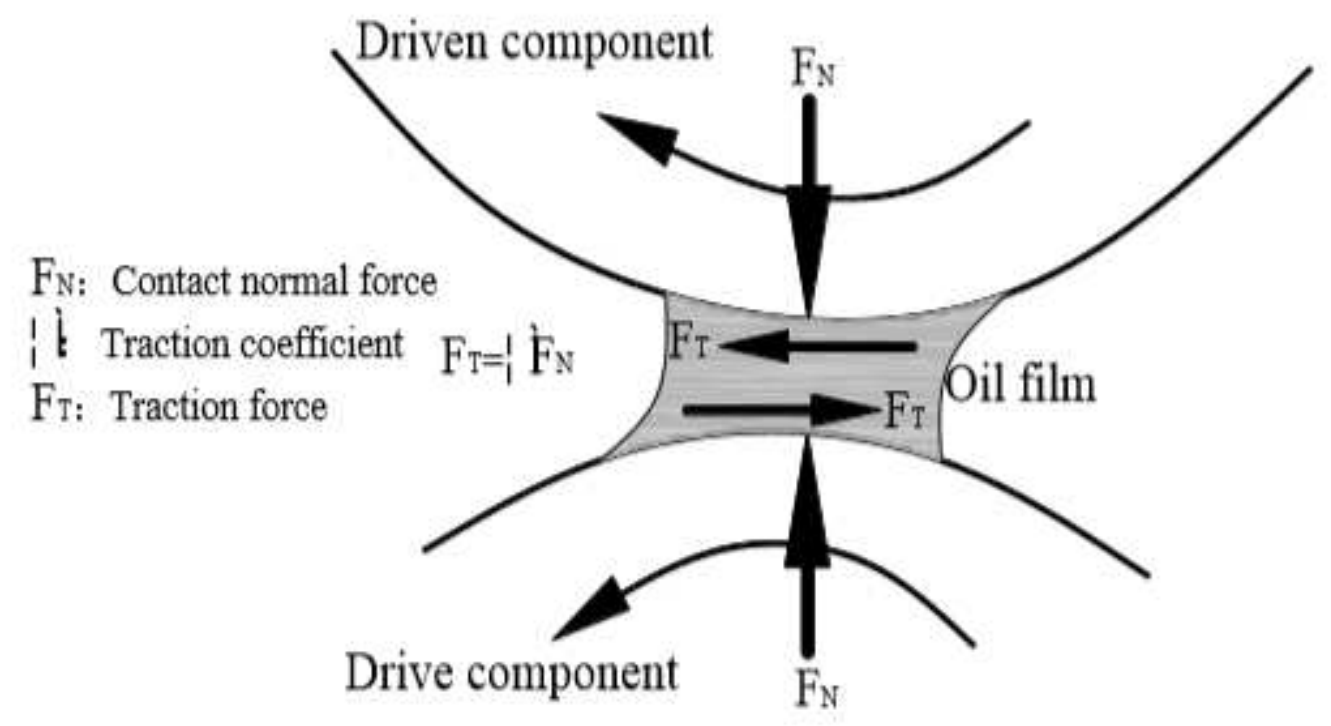

Figure 1

The main principle of power transmitting of t-CVT

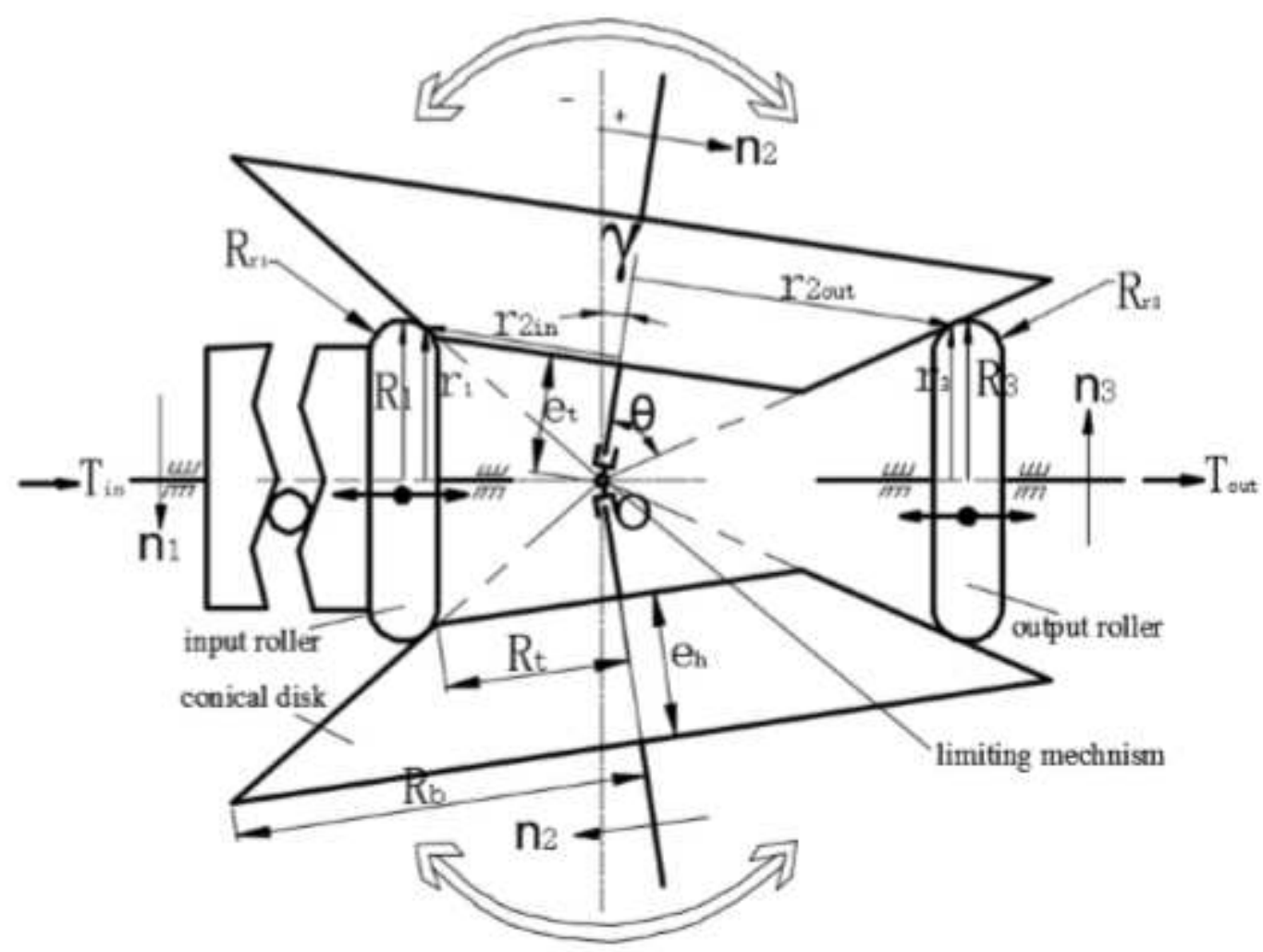

Figure 2 


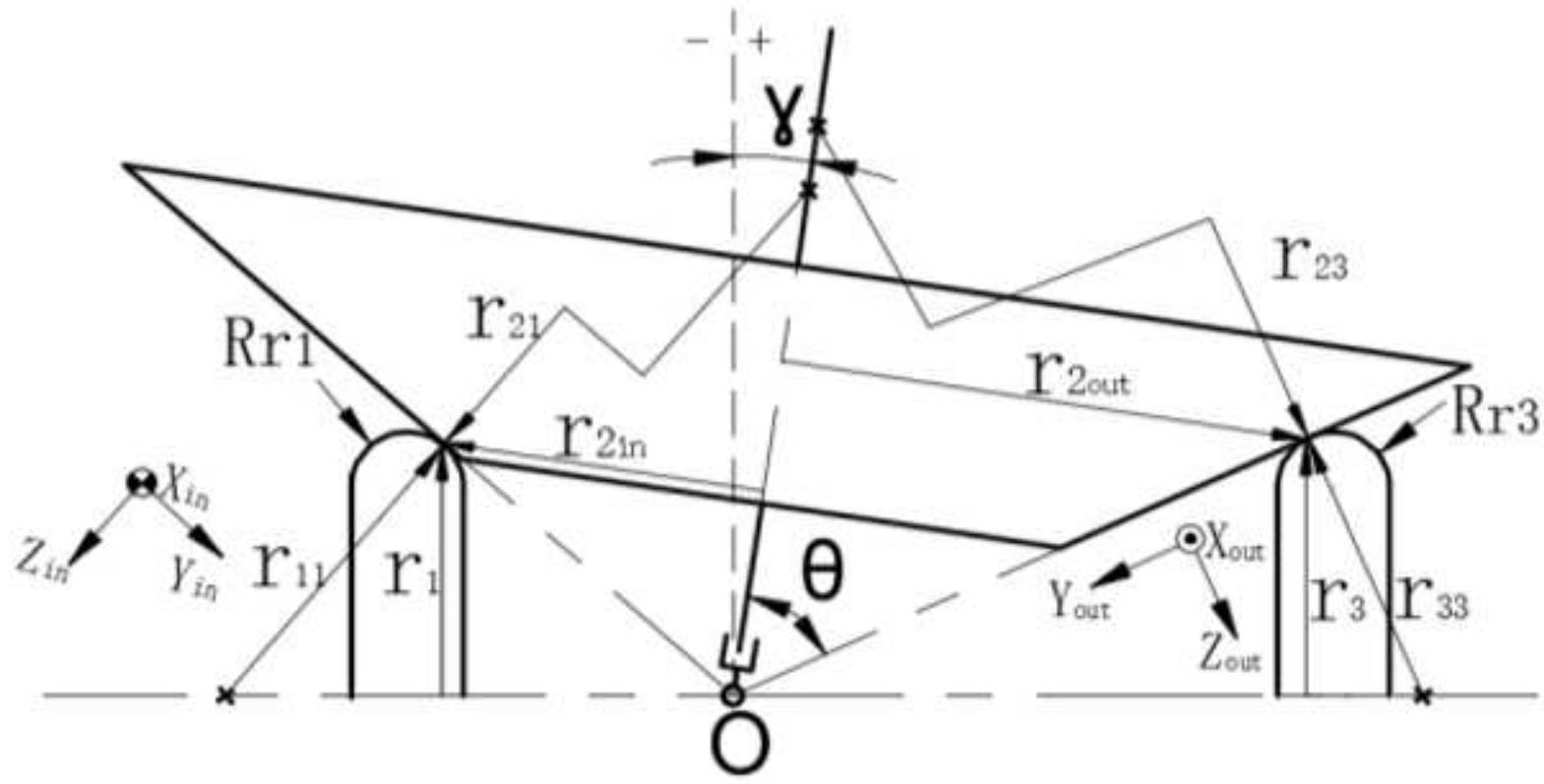

Figure 3

The reference frames and principal curvature radii for the rollers and conical disks 


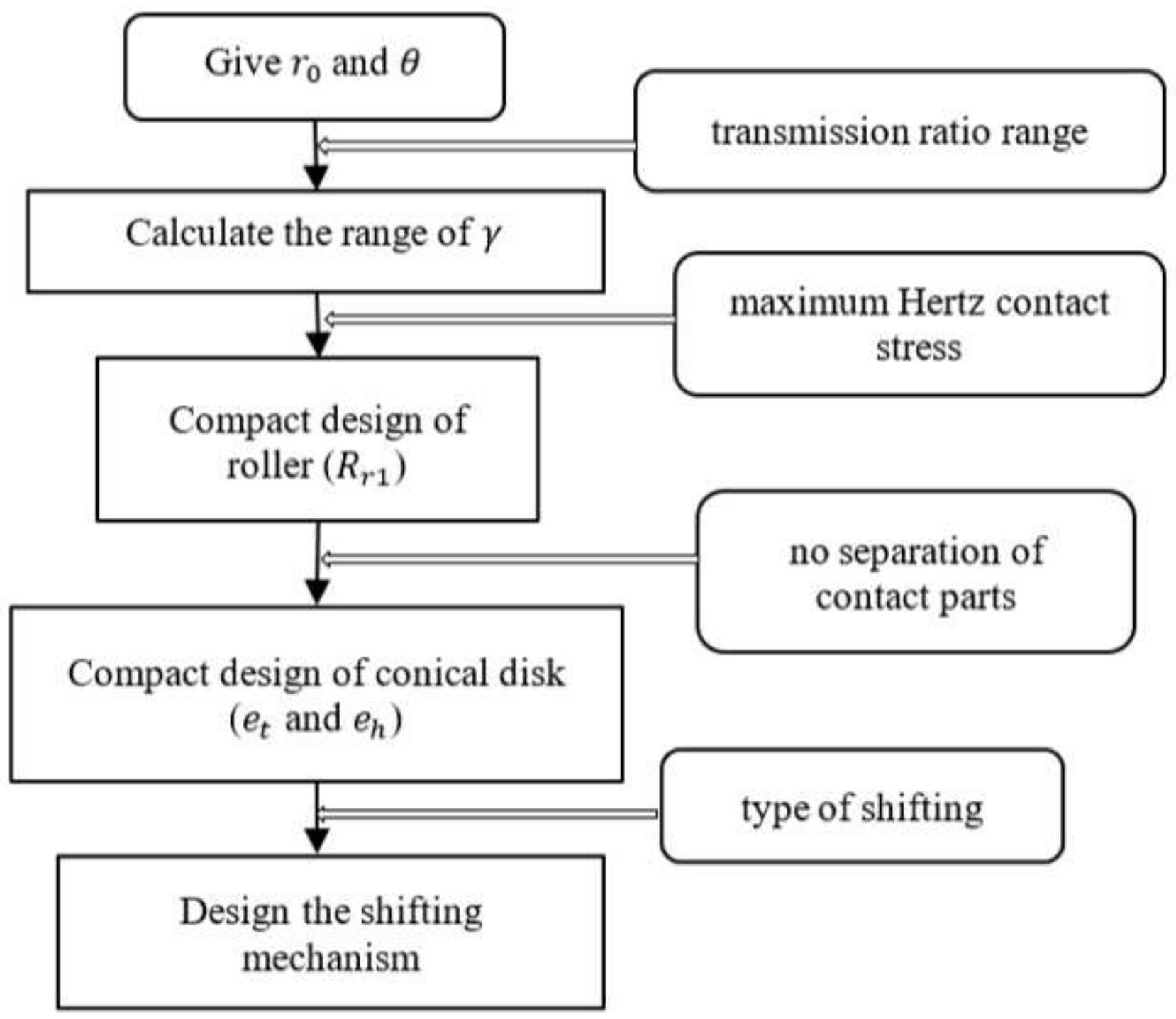

Figure 4

The design flowchart of RC-CVT 


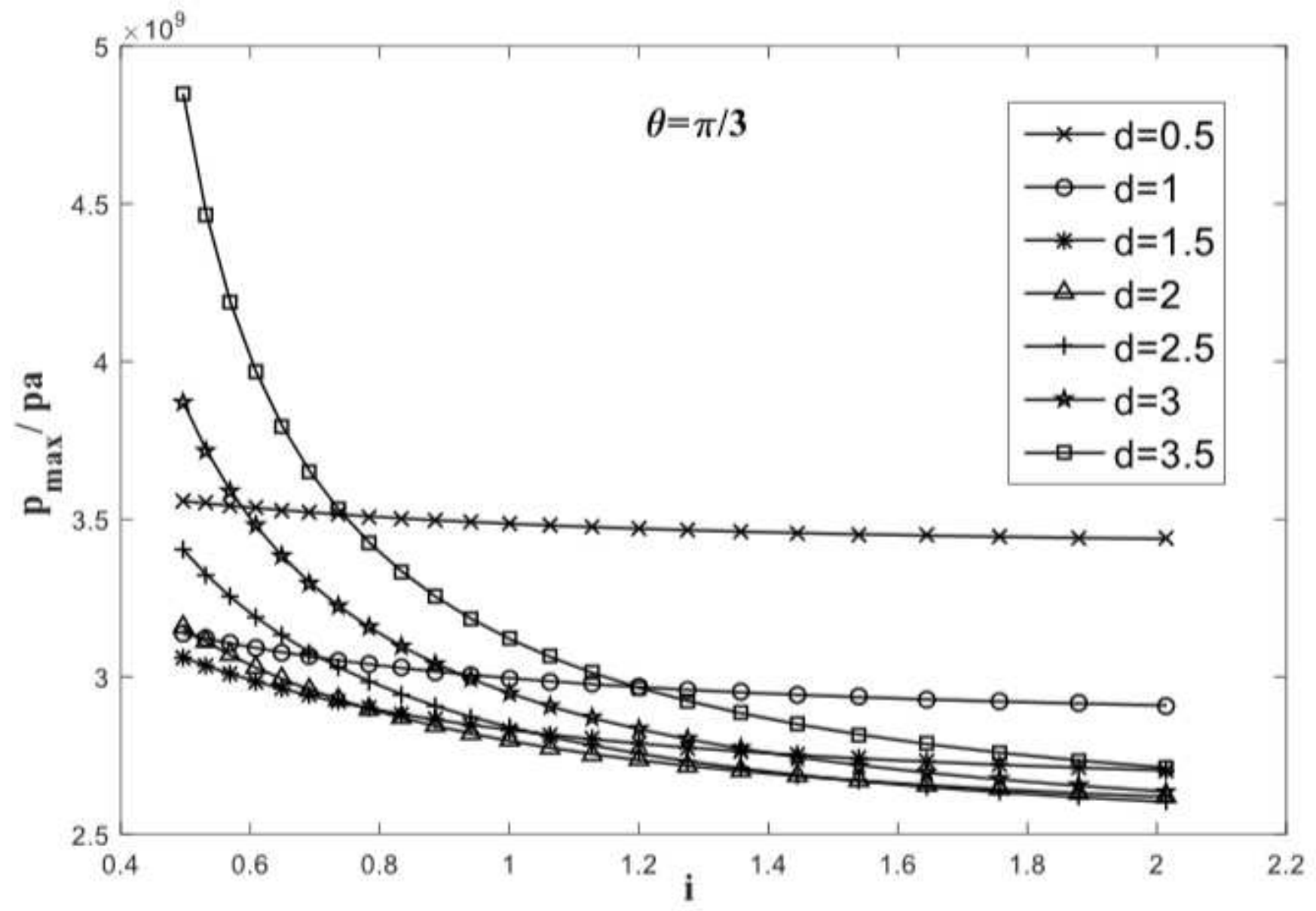

Figure 5

The design flowchart of RC-CVT 


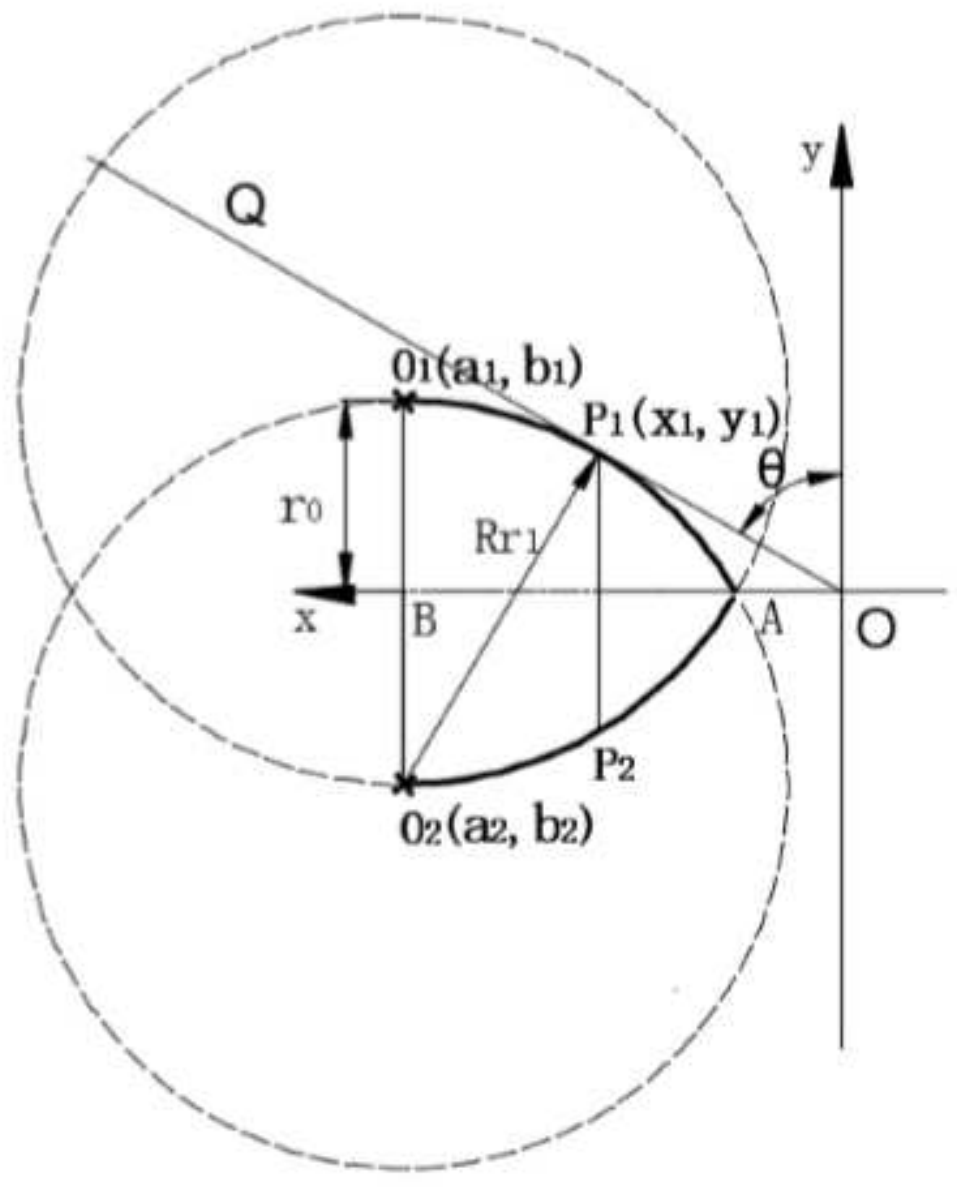

Figure 6

The designed curve of roller for RC-CVT 


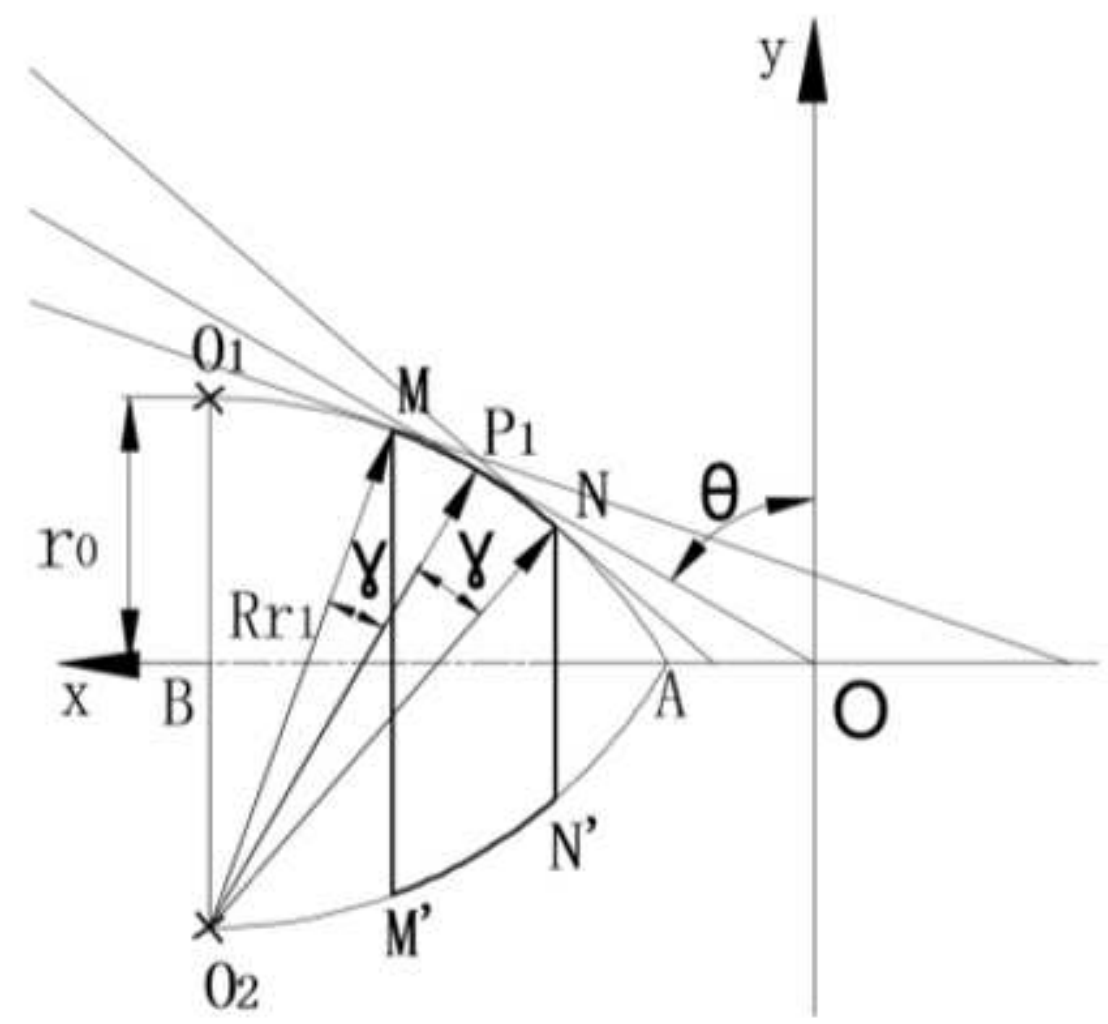

Figure 7

The compact design of roller for RC-CVT

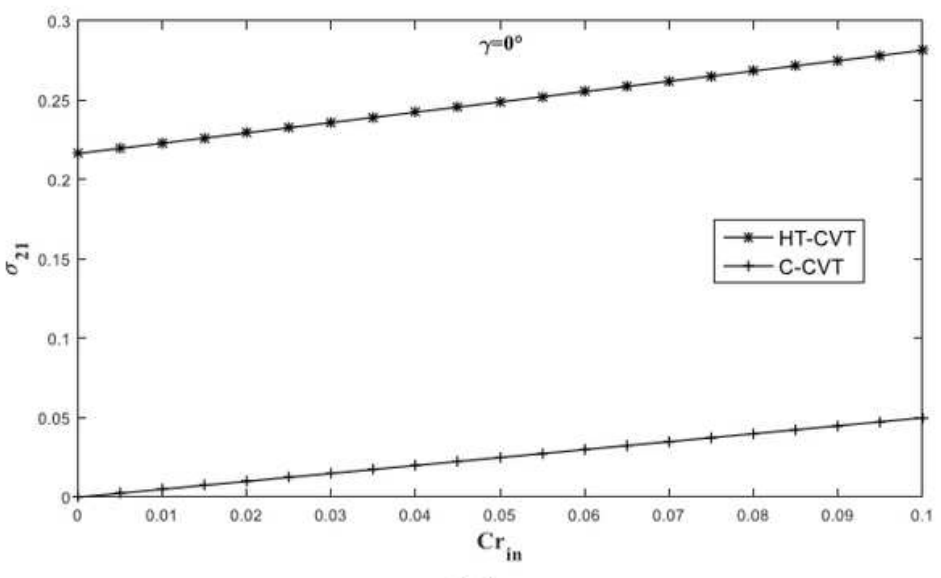

(a)

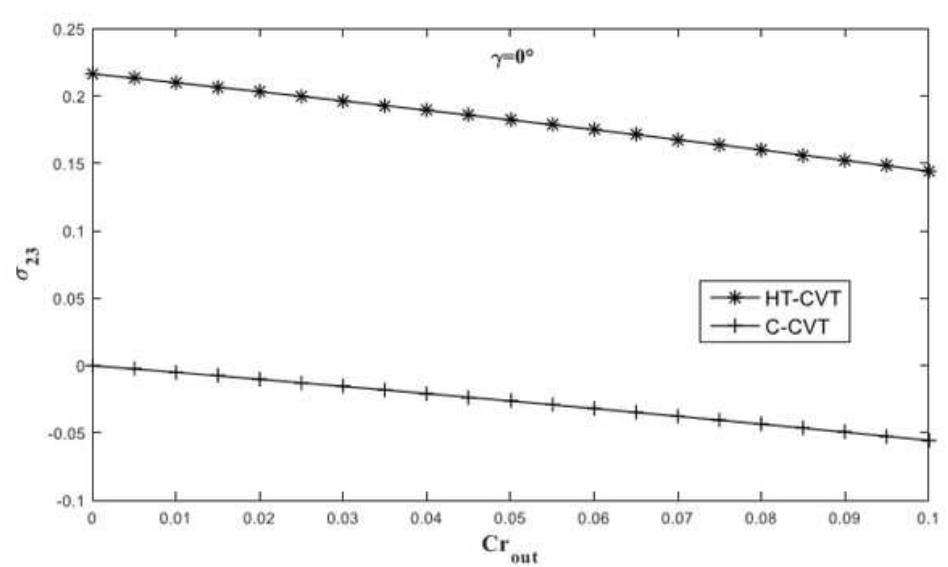

(b)

Figure 8

The practical spin ratio comparation between HT-CVT and RC-CVT: (a) The practical spin ratio at input contact; (b) The practical spin ratio at output contact 


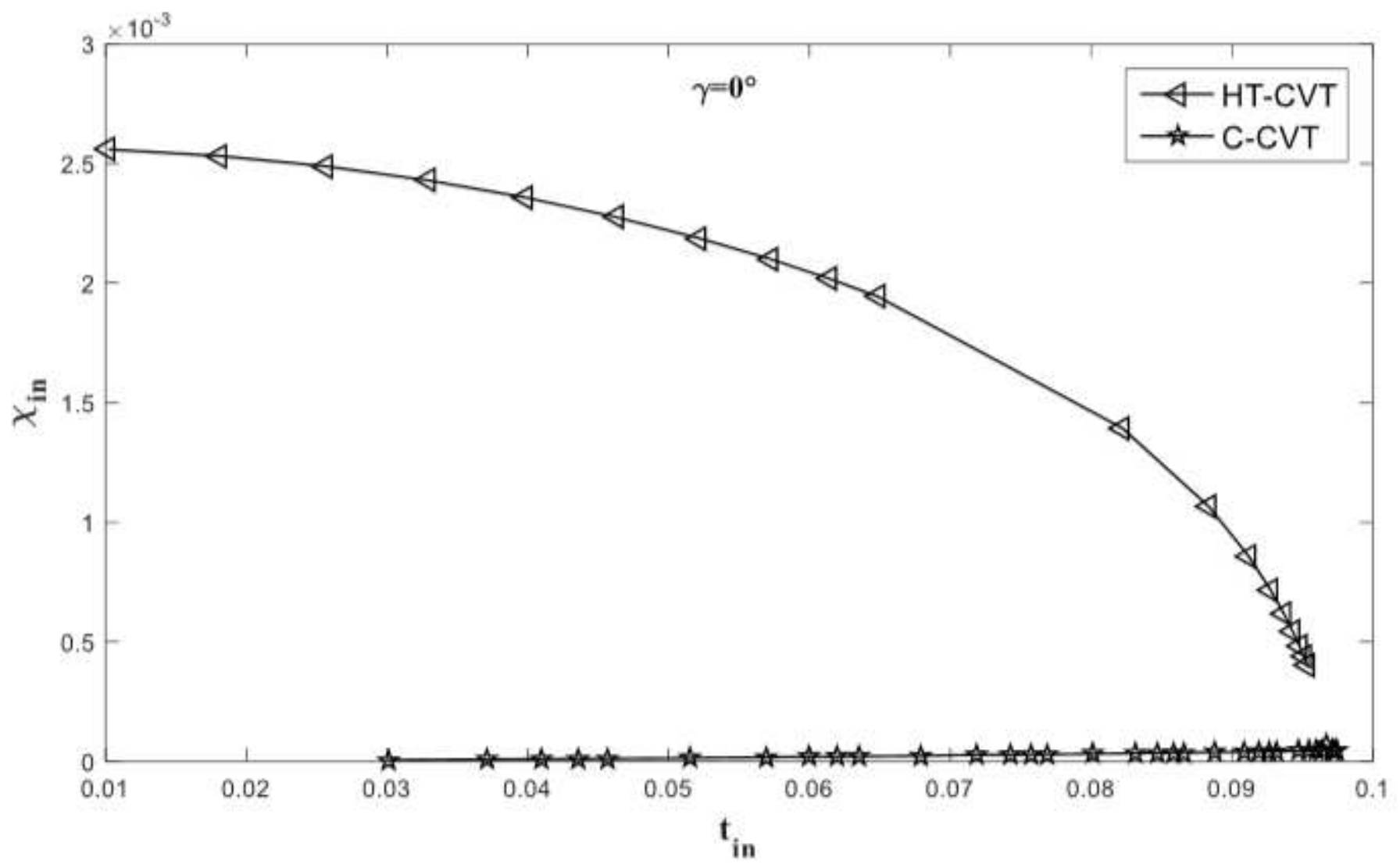

Figure 9

The spin moment $\chi_{-}$in as a function of input traction coefficient $t \_i n$ 


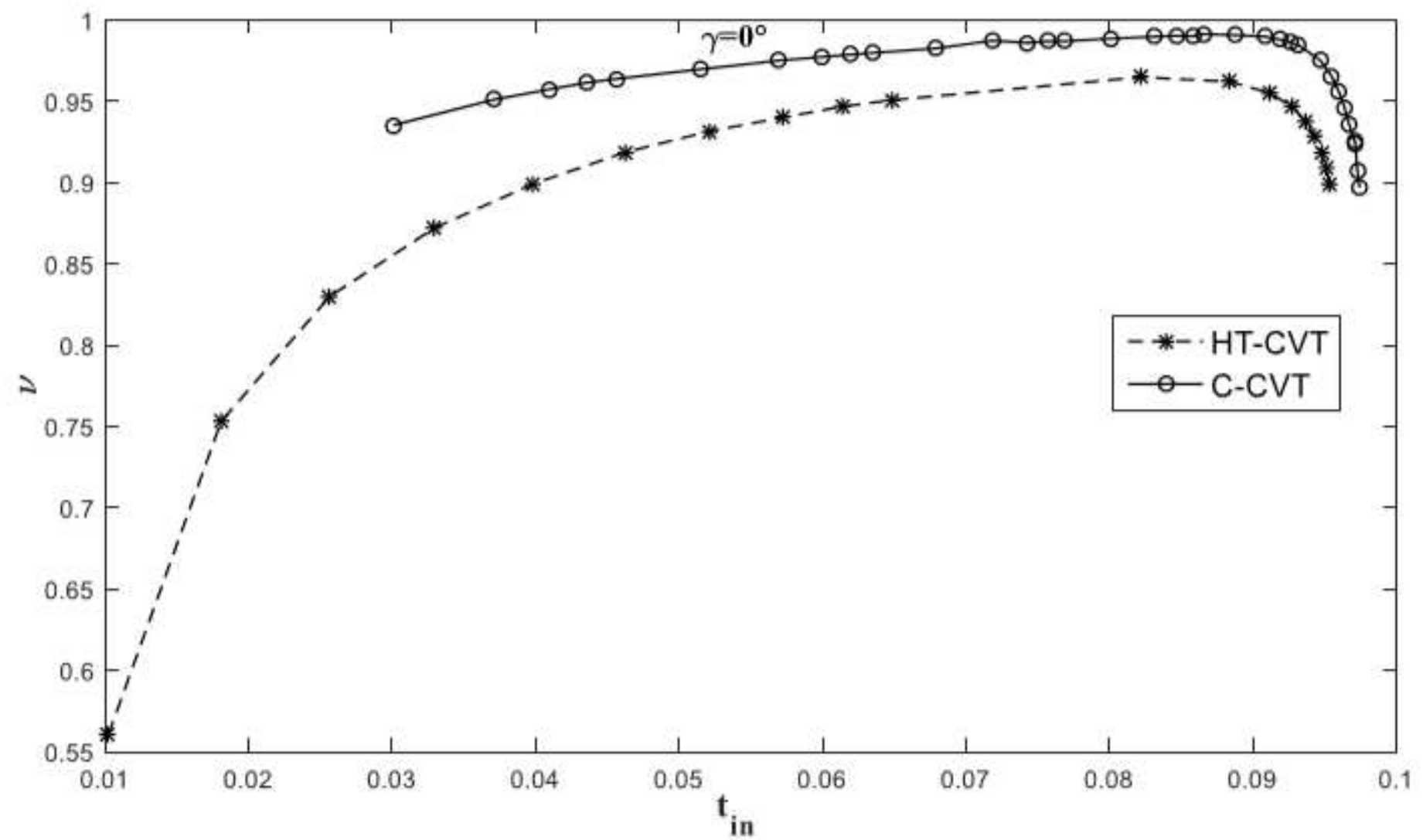

Figure 10

The efficiency of the CVTs as a function of the input traction coefficient t_in 


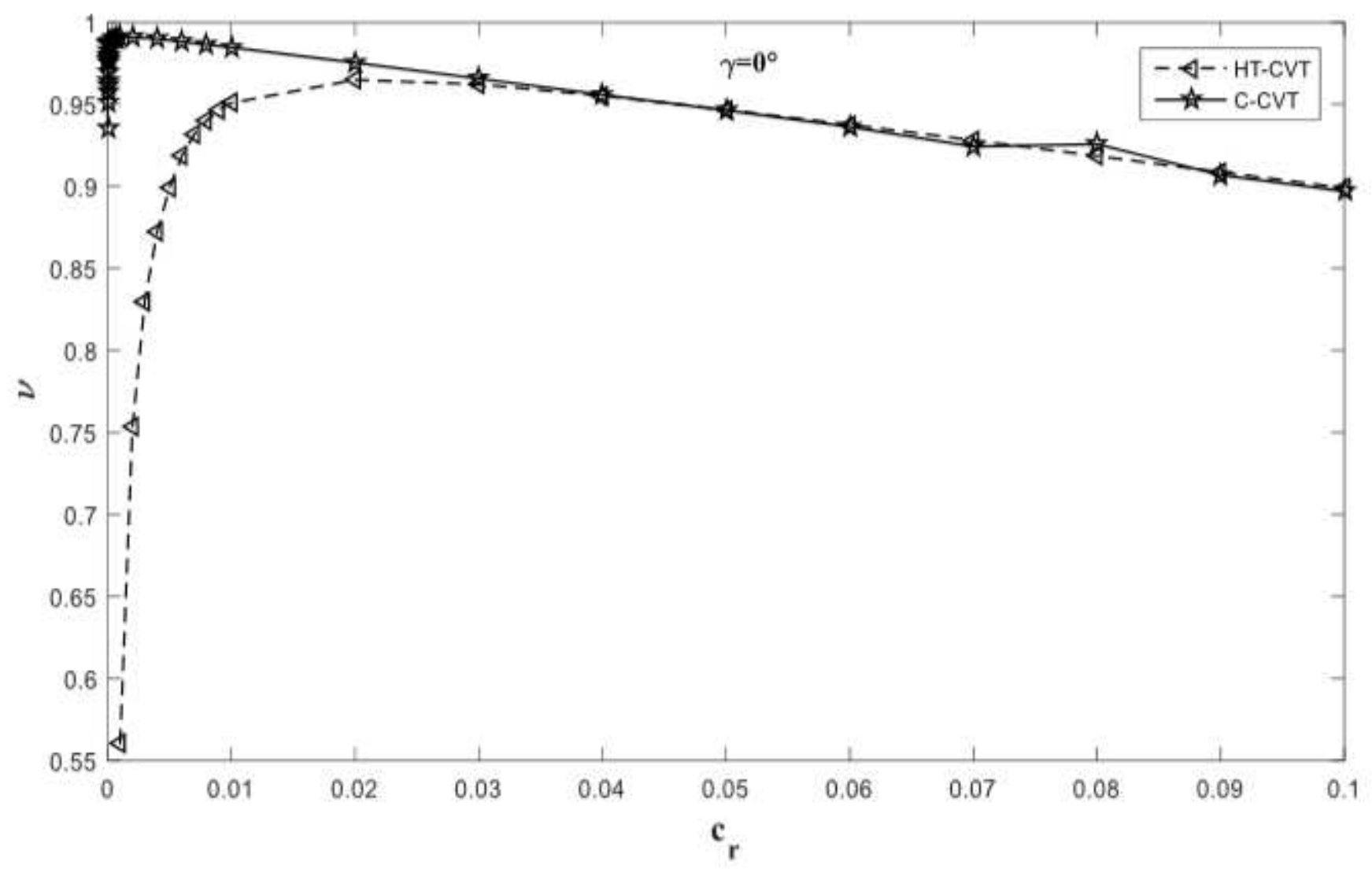

Figure 11

The efficiency of the CVTs as a function of the creep coefficient $C_{-} r$ 


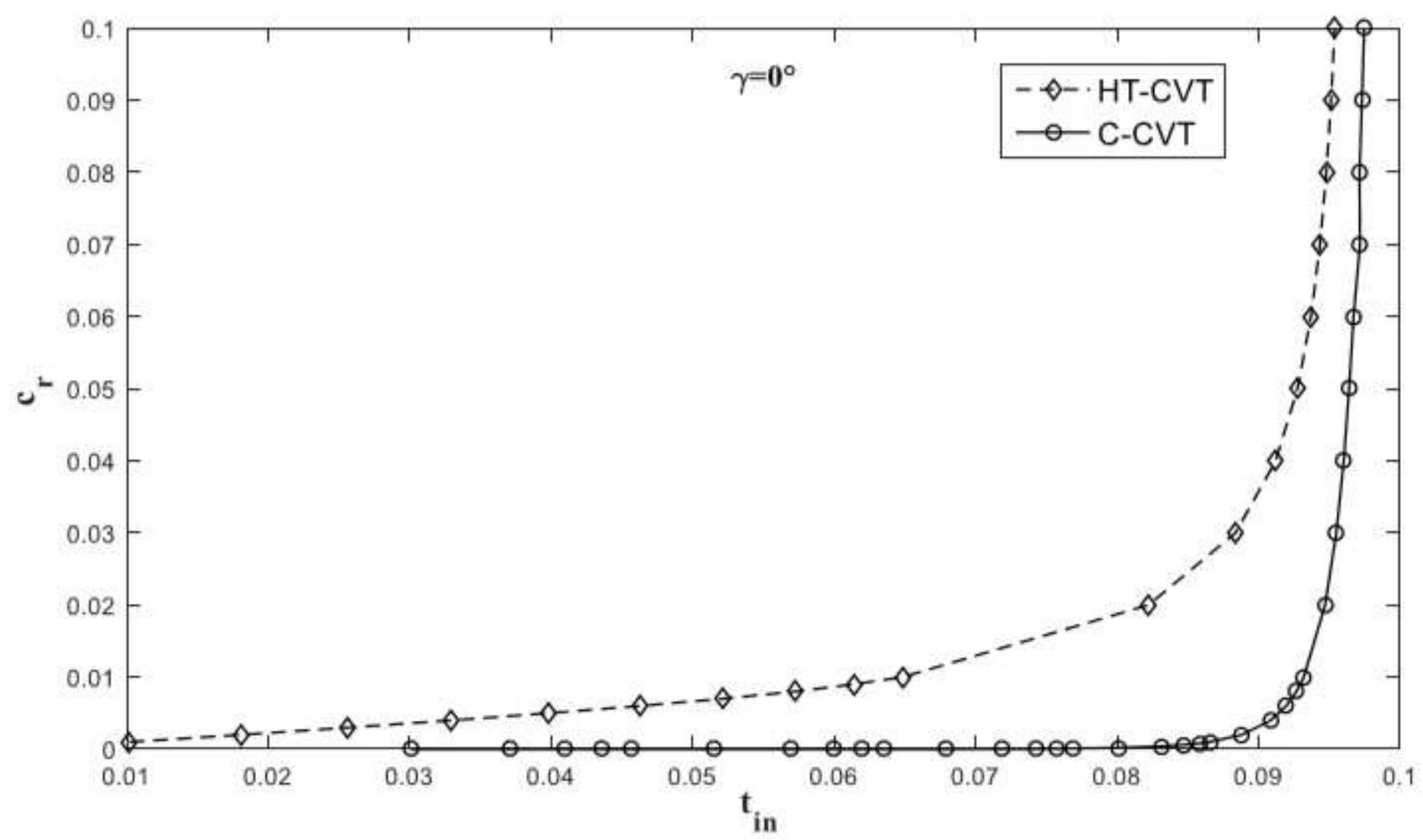

Figure 12

The relationship between t_in and C_r of HT-CVT and RC-CVT 